\title{
Association of School Environment and After-School Physical Activity with Health-Related Physical Fitness among Junior High School Students in Taiwan
}

\author{
Kai-Yang Lo ${ }^{1}$, Min-Chen $\mathrm{Wu}^{2}$, Shu-Chin Tung ${ }^{3}$, City C. Hsieh ${ }^{4}$, Hsueh-Hua Yao ${ }^{5}$ \\ and Chien-Chang $\mathrm{Ho}^{6, *}$ \\ 1 Center for General Education, National Sun Yat-sen University, Kaohsiung City 804, Taiwan; \\ luosun@ms25.hinet.net \\ 2 Office of Physical Education, Chung Yuan Christian University, Taoyuan City 320, Taiwan; \\ minchenwu.cycu@gmail.com \\ 3 Department of Health and Leisure Management, Yuanpei University of Medical Technology, \\ Hsinchu City 300, Taiwan; sctun@mail.ypu.edu.tw \\ 4 Department of Physical Education, National Tsing Hua University, Hsinchu City 300, Taiwan; \\ chsieh@mail.nhcue.edu.tw \\ 5 Department of Radiological Technology, Yuanpei University of Medical Technology, Hsinchu City 300, \\ Taiwan; yaohsuehhua@gmail.com \\ 6 Department of Physical Education, Fu Jen Catholic University, New Taipei City 242, Taiwan \\ * Correspondence: 093703@mail.fju.edu.tw; Tel.: +886-2-2905-3386; Fax: +886-2-2905-2380
}

Academic Editor: Derek Clements-Croome

Received: 11 August 2016; Accepted: 11 January 2017; Published: 15 January 2017

\begin{abstract}
The relationship between students' school environment and exercise habits is complex, and is affected by numerous factors. However, the few studies that have been conducted on this relationship have reported inconsistent results, especially regarding Taiwanese students. We conducted this cross-sectional study to investigate the association of school environment and after-school physical activity with health-related physical fitness in Taiwanese adolescents. Data were drawn from a national survey conducted by the Ministry of Education in Taiwan in 2008 of health-related physical fitness measurements among junior high school students (649,442 total) in grades seven to nine. School environment (level of urbanization, school size, presence of sports field or gymnasium) and after-school physical activity were assessed for their association with adolescents' physical fitness measurements (body mass index (BMI), bent-leg sit-ups, 800-/1600-m run, sit-and-reach, standing long jump). Urban boys and girls perform significantly better in muscle strength and endurance, cardiorespiratory endurance, flexibility, and explosive power; girls from rural areas exhibited significantly worse scores in body composition. Boys from large-size schools performed the worst in cardiorespiratory endurance, flexibility, and explosive power; whereas girls from large-size schools performed the worst in muscle strength, muscle endurance, and explosive power, but had the best score for body composition. However, the differences in body composition of boys from large-, medium-, and small- size schools did not reach a statistically significant level. Adolescents of both genders in schools with a sports field or gymnasium exhibited significantly better in muscle strength and endurance, cardiorespiratory endurance, and explosive power. Boys in schools with a sports field or gymnasium had significantly better body composition; girls in schools with sports field or gymnasium differed significantly in flexibility. Adolescents of both genders who participated in physical activity after school had significantly better body composition, cardiorespiratory endurance, and flexibility. Boys who participated in physical activity after school significantly differed in explosive power, whereas girls who participated in physical activity after school exhibited significantly better flexibility. Thus, the current study demonstrated that some factors, including urbanization (school location in rural or urban areas), school size, school facility provision (school with or without sports fields or gymnasiums), and after-school physical activity
\end{abstract}


participation are more important than others in shaping adolescents' physical fitness in Taiwan; meanwhile, these association patterns differed by gender.

Keywords: school environment; after-school physical activity; health-related physical fitness; adolescent

\section{Introduction}

Lack of regular physical activity is a well-documented risk factor for cardiovascular disease, hypertension, high cholesterol, obesity, and other chronic diseases [1-3]. Studies conducted in Taiwan have suggested that adverse health conditions may originate in childhood and adolescence $[4,5]$. The physical activity of adolescents is affected by a variety of factors, including age, gender, self-esteem, residential area, peer influences, parenting styles, their family's socioeconomic status, and ethnic disparities [6].

Physical fitness is interpreted as a measure of the ability to perform physical activity that integrates the majority of bodily functions, and its components are divided into those related to health and those related to athletic skill [7]. Several studies have demonstrated that more active school-age youth have better cardiorespiratory endurance, muscle strength, muscular endurance, flexibility, and body composition than their less active counterparts [8-10]. These findings suggest a link between physical activity and health-related physical fitness. Therefore, physical fitness is an important issue for the health promoting school programs implemented by the Taiwan Ministry of Education. The Taiwan Ministry of Education conducted a series of nationwide surveys that evaluated the health-related physical fitness of students in elementary, junior, and high schools [11,12]. The results of the survey provide a preliminary reference regarding the health-related physical fitness of children and adolescents in Taiwan. Adolescents spend considerable quantities of time at school, and it is therefore essential to understand what opportunities they are given for school-based physical activity. To improve the physical fitness of school-age children and adolescents, the Taiwan Ministry of Education recommended that students exercise three times per week for $30 \mathrm{~min}$, raising their heart rate to $130 \mathrm{bpm}$. Liu and Hsieh [13] emphasized a more practical approach involving accumulating intermittent physical activity for more than 10 min per time multiple times throughout the day. To monitor and improve adolescents' physical function and health status, a national recommendation on the health-related physical fitness of Taiwanese students must be established that can be applied in counseling and education.

Chen et al. [14] investigated the prevalence of overweight and obesity in nationally representative samples of 6-18-year-olds, using the National Physical Fitness Survey (NPFS) conducted by the National Council on Physical Fitness and Sports in Taiwan in 1999, repeated in 2001. In 2007, the NPFS offered another opportunity to study the interrelationships between weight, hypertension, and health-related physical fitness measurements as early indicators of ill health.

Evidence indicates that sufficient school facilities for physical activity-such as sports fields and gymnasiums - promote physical activity in adolescents [15]. Thus, the lower levels of physical activity of adolescents enrolled in large-size schools might be related to poor facility provision. However, no previous study in Taiwan has investigated how students' physical activity or fitness is related to school size. In addition, the after-school period is considered a "critical interval" that defines the propensity of adolescents for physical activity [16]. The after-school period may represent significant opportunities for educators and researchers to modify exercise habits and enhance physical activity for adolescents reported by Pelclová et al. [17]. In this study, we performed a cross-sectional investigation of environmental variables and health-related physical fitness, measuring five physical fitness indicators in Taiwanese adolescents aged 13-15 years. The aim of this study was to gather essential information for use in health policy reform concerning the health-related physical fitness of Taiwan's adolescents. 


\section{Materials and Methods}

\subsection{Participants}

The data consisted of 649,442 Taiwanese adolescents $(N=312,365$ girls and $N=337,077$ boys $)$ in the seventh to ninth grades of junior high school, and were collected between February and June 2008. Students with a disability were excluded, because of the difficulty in the conducting health-related physical fitness measurements of these students. The measurements were made by school physical education teachers and nurses, all of whom had attended a regional training seminar and passed a certification test on standardized procedures. The protocols to be used were described in detail in a technical manual provided to all the junior high schools and participating teachers and nurses.

Both parents and students were informed about the aims and procedures of this study, and written informed consent was obtained from parents or guardians, as well as students providing their assent. The study was performed in accordance with the Helsinki Declaration of 1975 with regard to the conduct of clinical research, and was approved by the Ethical Committee of Ministry of Education in Taiwan (SAHIARC-MOE 104004).

\subsection{Data Collection}

The investigating variables—school environment (urbanization, school size, and school facility provision) and after-school physical activity-are described as follows.

\subsubsection{Urbanization}

Urbanization was determined according to the urban-rural classification established by the Ministry of Education in Taiwan, in which population density, age composition, mobility, economic activity and family income, educational level, and health and sanitation facilities were considered. The urban group consists of adolescents whose schools are located in the three largest cities in Taiwan (i.e., Taipei, Taichung, and Kaohsiung Cities). All non-urban adolescents studying in the schools in remote rural areas are classified as the rural group.

\subsubsection{School Size}

School size was determined based on the number of classes (i.e., the number of administrative groups of students) in each of the Taiwanese junior high schools for the grades seven to nine. A larger-size school was defined as one with 49 or more classes, a medium-size school as one with 13-48 classes, and a small-size school as one with fewer than 12 classes. The number of students for each class in junior high school ranged from 20 to 34, according to guidelines for class size in Taiwan.

\subsubsection{School Facility Provision}

School facility provision was determined according to whether schools had a sports field and gymnasium, and was based on the 2008 annual report of physical education statistics.

\subsubsection{After-School Physical Activity Participation}

In contrast with physical education classes at school, after-school physical activity was available to students with access to out-of-school sports facilities or home exercise equipment. After-school physical activity participation was determined based on whether the school's students exercised after school. The frequency of after-school physical activity participation was defined as being more than one time per week. The recommended target for time spent in moderate to vigorous leisure-time physical activity was over $30 \mathrm{~min}$ after the normal school day. The Taiwan Ministry of Education recommended that the exercise frequency for students is three times per week with an intensity of heart rate of $130 \mathrm{bpm}$ for $30 \mathrm{~min}$ per exercise session (i.e., moderate to vigorous exercise intensity). To fulfill the recommendation, each student in this study was required to participate in a physical 
education class twice per week during school days in addition to engaging in after-school physical activity more than once. The data used were determined using the 2008 annual report of physical education statistics.

\subsection{Health-Related Physical Fitness Measurements}

The following five health-related physical fitness measurements were taken according to the governmental guideline indicated on the official website of the Taiwan Ministry of Education [18]: body composition (body mass index, BMI), muscle strength and endurance (bent-leg sit-ups), explosive power (standing long jump), flexibility (sit-and-reach), and cardiorespiratory endurance (800-/1600-m run). The measurements were performed by school physical education teachers and nurses with the appropriate training. Inspectors from the Taiwan Ministry of Education and universities visited each site to supervise progress and adherence to standards.

\subsubsection{Body Composition}

Body composition was measured using BMI. Participants took off their shoes and wore light clothes prior to the measurement of their height and weight. BMI was calculated as their weight in kilograms divided by the square of their height in meters $\left(\mathrm{kg} / \mathrm{m}^{2}\right)$. Weight and height were measured using a stadiometer and a digital or balance beam scale. All instruments were validated and approved by the Taiwan Bureau of Standards, Metrology, and Inspection.

\subsubsection{Muscle Strength and Endurance}

Muscle strength and endurance was measured as the number of bent-leg sit-ups a student was able to perform within $1 \mathrm{~min}$. Each participant lay supine on a mat, with knees bent at right angles and their hands crossed on the chest. A technician held the participant's ankles firmly for support and conducted the count. The participant's elbows had to touch the corresponding knee (i.e., left touch left, etc.). After each upward movement, the shoulders returned to touch the mat, but touching the mat with the head was not required.

\subsubsection{Explosive Power}

Explosive power was measured through a standing long jump. The participants stood on a starting line and were instructed to jump forward with both feet as far as possible. They were free to use their arms and legs for countermovement. The distance from the starting line to the heel of the foot closest to the line was recorded. The longer distance from two attempts was recorded in centimeters.

\subsubsection{Flexibility}

Flexibility was measured using the standard sit-and-reach test, scored by the most distant point reached with the fingertips on a ruler. The device used had a measuring scale positioned such that reaching the feet with the fingertips recorded a distance of $25 \mathrm{~cm}$. Before the test, shoes were removed and participants were instructed to slowly reach forward as far as possible with their knees fully extended. The longer distance from two attempts was recorded in centimeters.

\subsubsection{Cardiorespiratory Endurance}

Cardiorespiratory endurance was measured through an 800-m (for girls) or 1600-m (for boys) run. Adequate warm-up exercise was performed before the test. The participants ran in groups of six to eight from behind a starting line, and were instructed to try to keep a steady speed, finishing the run as quickly as possible. 


\subsection{Statistical Analysis}

All data were processed using Statistical Package for the Social Science (SPSS) software (SPSS 22.0 version for Windows, SPSS Inc., Chicago, IL, USA). Descriptive statistics including means and standard deviations (SDs) were calculated for all variables. The effects of urbanization, school size, school facility provision, and after-school physical activity on health-related physical fitness measurements were evaluated using the Student's $t$ test or analysis of variance, with the significance level being set at 0.05 . When a significant $p$ value was found $(p<0.05)$, Tukey's post hoc test was performed to determine the differences between the mean pairs. All values in tables are displayed as the means $\pm S D$.

\section{Results}

\subsection{Participant Demographics}

Table 1 presents the participant demographics of the sample, including a breakdown of their age and gender. A total of 312,365 girls and 337,077 boys from the seventh through ninth grades in junior high schools participated in this study.

Table 1. Demographics of study participants.

\begin{tabular}{cccc}
\hline Age (Years) & Total & Boys & Girls \\
\hline 13 & 226,733 & 118,091 & 108,642 \\
14 & 219,343 & 113,313 & 106,030 \\
15 & 203,366 & 105,673 & 97,693 \\
Total & 649,442 & 337,077 & 312,365 \\
\hline
\end{tabular}

\subsection{Association of Urbanization and School Size with Health-Related Physical Fitness}

Of the participants, $7.08 \%$ of the boys $(N=21,101)$ and $6.87 \%$ of the girls $(N=19,249)$ lived in rural areas (Table 2). The BMI values of the boys in rural areas were comparable to those of the boys in urban areas. Girls who attended rural schools, however, had significantly higher BMI values than those who attended urban schools $\left(20.05 \pm 2.96\right.$ vs. $\left.19.85 \pm 2.91 \mathrm{~kg} / \mathrm{m}^{2}, p<0.05\right)$. No significant difference was found in the BMI of boys attending schools of varying size (Table 2). Girls attending small-size schools, however, were discovered to have a significantly higher BMI $(p<0.05)$-a trend comparable to that for schools in rural and urban areas.

Table 2. Health-related physical fitness measurements for participants, grouped according to urbanization and school size.

\begin{tabular}{|c|c|c|c|c|c|}
\hline & BMI & Bent-Leg Sit-Ups & 800/1600-m Run & Sit-and-Reach & Standing Long Jump \\
\hline \multicolumn{6}{|l|}{ Boys } \\
\hline Rural $(N=21,101)$ & $20.7 \pm 3.7$ & $37.4 \pm 10.3 *$ & $581 \pm 141 *$ & $26.0 \pm 9.5^{*}$ & $185 \pm 32 *$ \\
\hline \multicolumn{6}{|l|}{ Girls } \\
\hline Rural $(N=19,249)$ & $20.05 \pm 2.96^{*}$ & $29.9 \pm 8.8^{*}$ & $305 \pm 68^{*}$ & $29.2 \pm 9.7^{*}$ & $145 \pm 26^{*}$ \\
\hline Urban $(N=260,758)$ & $19.85 \pm 2.91$ & $29.4 \pm 8.7$ & $303 \pm 63$ & $30.2 \pm 9.7$ & $144 \pm 25$ \\
\hline Large-size school $(N=137,484)$ & $20.7 \pm 3.6$ & $36.8 \pm 9.9 * *$ & $598 \pm 136^{* *,+}$ & $25.6 \pm 9.3^{* *,+}$ & $182 \pm 32 * *,+$ \\
\hline Medium-size school $(N=139,090)$ & $20.7 \pm 3.7$ & $36.4 \pm 10.2 \ddagger$ & $592 \pm 138$ & $26.0 \pm 9.4 \ddagger$ & $183 \pm 32$ \\
\hline Small-size school $(N=21,074)$ & $20.7 \pm 3.7$ & $36.9 \pm 10.4$ & $572 \pm 142$ & $26.3 \pm 9.5$ & $184 \pm 32$ \\
\hline \multicolumn{6}{|l|}{ Girls } \\
\hline Large-size school $(N=130,184)$ & $19.8 \pm 2.9^{* *, \dagger}$ & $29.7 \pm 8.6^{* *,+}$ & $304 \pm 63 * *$ & $30.2 \pm 9.7^{\dagger}$ & $144 \pm 25^{* *, \dagger}$ \\
\hline
\end{tabular}

Notes: $\mathrm{BMI}=$ body mass index; $\mathrm{SD}=$ standard deviation; Values are expressed as mean $\pm \mathrm{SD}$; Significant difference between rural and urban schools among same-gender adolescents $(p<0.05)$; ** Significant difference between large- and medium-sized schools among same-gender adolescents $(p<0.05) ;{ }^{\dagger}$ Significant difference between large- and small-size schools among same-gender adolescents $(p<0.05)$; ${ }^{\ddagger}$ Significant difference between medium- and small-size schools among same-gender adolescents $(p<0.05)$. 
Significant differences in physical fitness between small and large-size schools for both boys and girls were discovered. Boys from small-size schools were also found to have better performance at the 800-/1600-m run, sit-and-reach, and standing long jump than did those from large-size schools. Girls from small-size schools had better performance at the standing long jump, but could not reach as far in the sit-and-reach as girls from large-size schools did.

\subsection{Association of School Facility Provision and After-School Physical Activity with Health-Related Physical Fitness}

Of the junior high schools investigated in this study, approximately $70 \%$ and $90 \%$ had a sports field or indoor gymnasium, respectively (Table 3). Boys in schools with access to a sports field performed significantly better in the physical fitness tests than those without access to a sports field, except for the flexibility test. Girls in schools with access to a sports field had significantly higher muscular endurance, explosive power, and cardiorespiratory ability than did those in schools without, but significantly lower flexibility and similar BMI values. Similar results were obtained from boys and girls in the indoor gymnasium.

Approximately one-quarter (26\%) of students took exercise after school (Table 3). The BMI and flexibility of adolescents of both genders who participated in exercise after school were better than those of adolescents who did not. The other three items relevant to physical fitness had inconsistent results between genders.

Table 3. Health-related physical fitness measurements for participants, grouped according to their schools' facilities and whether they exercise after school.

\begin{tabular}{|c|c|c|c|c|c|}
\hline & BMI & $\begin{array}{l}\text { Bent-Leg } \\
\text { Sit-Ups }\end{array}$ & $\begin{array}{l}\text { 800/1600-m } \\
\text { Run }\end{array}$ & Sit-and-Reach & $\begin{array}{l}\text { Standing Long } \\
\text { Jump }\end{array}$ \\
\hline \multicolumn{6}{|l|}{ Boys } \\
\hline With sport field $(N=268,863)$ & $20.68 \pm 3.65 *$ & $36.7 \pm 10.1 *$ & $593 \pm 136^{*}$ & $25.8 \pm 9.4$ & $183 \pm 32 *$ \\
\hline \multicolumn{6}{|l|}{ Girls } \\
\hline With sport field $(N=252,201)$ & $19.9 \pm 2.9$ & $29.5 \pm 8.7^{*}$ & $303 \pm 64 *$ & $30.1 \pm 9.7^{*}$ & $144.3 \pm 25.0 *$ \\
\hline With gymnasium $(N=201,726)$ & $20.67 \pm 3.63^{* *}$ & $36.7 \pm 10.1^{* *}$ & $592 \pm 136$ ** & $25.9 \pm 9.4$ & $183 \pm 32 * *$ \\
\hline Without gymnasium $(N=90,565)$ & $20.73 \pm 3.68$ & $36.5 \pm 10.0$ & $597 \pm 140$ & $25.8 \pm 9.3$ & $182 \pm 32$ \\
\hline \multicolumn{6}{|l|}{ Girls } \\
\hline With gymnasium $(N=192,148)$ & $19.9 \pm 2.9$ & $29.5 \pm 8.8^{* *}$ & $303 \pm 64^{* *}$ & $30.2 \pm 9.7^{* *}$ & $145 \pm 25^{* *}$ \\
\hline Without gymnasium $(N=82,432)$ & $19.9 \pm 2.9$ & $29.3 \pm 8.5$ & $304 \pm 64$ & $30.0 \pm 9.6$ & $143 \pm 25$ \\
\hline \multicolumn{6}{|l|}{ Girls } \\
\hline With physical activity after school $(N=72,186)$ & $19.8 \pm 2.9^{* * *}$ & $29.6 \pm 8.7^{* * *}$ & $304 \pm 66^{* * *}$ & $30.3 \pm 9.8^{* * *}$ & $144 \pm 26$ \\
\hline Without physical activity after school $(N=207,414)$ & $19.9 \pm 2.9$ & $29.4 \pm 8.7$ & $302 \pm 63$ & $30.1 \pm 9.7$ & $144 \pm 25$ \\
\hline
\end{tabular}

\section{Discussion}

From analysis of our data, we would first recommend the definition of national objectives for the health-related physical fitness of adolescents. Second, the conduction of a national surveillance study wherein the health-related physical fitness of adolescents is measured more precisely and consistently is suggested. Finally, the factors that may affect the health-related physical fitness of Taiwanese adolescents should be evaluated. This study recruited a total of 312,365 girls and 337,077 boys from the seventh through ninth grades of junior high schools in Taiwan. Of the students, $7.08 \%$ of boys and $6.87 \%$ of girls lived in rural areas. The BMI values of the boys living in rural areas were comparable to 
those of the boys living in urban areas. However, girls attending rural schools had significantly higher BMI values than those of girls attending urban schools $(p<0.05)$.

A great deal of effort was applied to comparing the physical fitness performance of rural and urban adolescents in Taiwan. We discovered that, for both genders, students in rural schools performed better in physical fitness tests than did those attending urban schools. These findings could be due to differences of age groups and gender differences. Students' physical activity levels decreased with age, and boys had higher levels of physical fitness than did girls $[4,19,20]$. This gender difference could be due to the different stages of physical and psychological development of the boys and girls, and the different gender expectations they face [21,22]. Boys in Taiwan are encouraged to participate in sports-related activities more often than girls are [23], and are more competent at physical activities $[19,20]$.

The current results are not consistent with those reported by Huang et al. [24], who indicated that no relationship exists between residential area and students' physical activity levels. Another study identified that urban adolescents have higher physical activity levels than rural adolescents [25], possibly because urban adolescents have more access to sport facilities and more choices for recreational and leisure activities. Students' age, sample sizes, different measures of physical fitness, and geographical locations may also be factors. Huang et al. [26] reported no significant difference in walking ability that may be improved physical fitness was found between adolescents from urban and rural areas. However, urban adolescents were found to have greater access to places suitable for physical fitness activities. Urban adolescents engaged in more physical activity after school, on holidays and weekends, and in total compared with rural adolescents.

Confirming the results of our study, Chen [27] reported that adolescents from rural areas had superior muscle strength and endurance relative to their counterparts in urban areas. Shen and Huang [28] demonstrated that larger living spaces and living in rural areas were related to higher levels of physical fitness and activity among elementary school-age youth. Chen et al. [29] discovered that adolescents who attended rural schools had higher muscular endurance and flexibility than those who attended urban schools. Their results showed that urban schools provide more playing spaces (i.e., 0.58 square feet per urban student vs. 0.20 square feet per rural student) and exercise equipment than in the rural schools. However, rural schools may have more total spaces (1.22 square feet per urban student vs. 5.09 square feet per rural student) than in the urban schools. Numerous studies have reported that greater space per student indicates a higher likelihood of a student being physically active $[28,30,31]$. Conflicting results have been reported with respect to the importance of residential area as a determinant of physical fitness and physical activity in adolescents $[4,30]$. Lower levels of physical activity in urban youth may be due to unsafe neighborhoods, limited access to parks and gyms, and poor facility provision [15,32].

Previous studies on the physical fitness of adolescents have used considerably smaller samples than that used this study. We can conclude that rural students in Taiwanese junior high schools exhibited superior physical fitness relative to students attending urban schools, regardless of gender, and that numerous factors were found to contribute to differences in physical activity. Gender differences were discovered to be significant for all schools, which is consistent with other results $[33,34]$, and indicates that girls from both rural and urban areas must be targeted for priority intervention programs. Schools could be crucial to increasing girls' physical activity levels if they provided adequate equipment and spaces for activity, and promoted equal gender participation.

Chen et al. [29] reported that urban girls had higher flexibility than did rural girls, which is related to higher muscular endurance; however, no difference was found in boys. The different types of activities boys and girls are encouraged to participate in might help explain why certain factors contributed to their physical fitness. Girls were discovered to be less likely to adopt an active lifestyle unless there was a motivating factor (such as weight loss) or a compelling factor (such as a doctor's orders). 
In this study, we discovered that boys attending schools with a sports field performed well in the indicators of physical fitness, except for flexibility, for which no significant difference was found. Girls attending schools with a sports field had significantly higher muscular endurance, explosive power, and cardiorespiratory ability, but significantly lower flexibility and similar BMI values. School gymnasium provision also contributed to higher physical fitness in both genders. Girls attending schools with a gymnasium had higher flexibility than did those attending schools without. Convenient before-school, in-school, after-school, or weekend access to student-only facilities including playgrounds, multipurpose rooms, and auditoriums, might be essential for the physical activity levels and fitness of adolescents.

One advantage to this study was that selection biases could be eliminated, because the data were from a national survey that was representative of general Taiwanese adolescents. However, several limitations needed to be considered. First, we could not demonstrate causal associations of school environment and after-school physical activity with health-related physical fitness, because this is only a cross-sectional study. Although cross-sectional design is important to understand the relationships between school environment, after-school physical activity, and health-related physical fitness, prospective studies exploring causal association are needed. Second, this study assessed body composition on the basis of BMI. Despite its extensive use to define obesity status, BMI is not an accurate measure of the proportion of fat and fat-free tissue in the body. Percentage of body fat should be considered, and precise measures of body fat should be obtained in future studies.

\section{Conclusions}

Our findings demonstrated that some factors, including urbanization (school location in rural or urban areas), school size, school facility provision (school with or without sports fields or gymnasiums), and after-school physical activity participation are more important than others in shaping adolescents' physical fitness in Taiwan; meanwhile, these association patterns differed by gender. Our results can provide valuable information to the Taiwanese government in designing the health promoting schools programs for improving the physical fitness and health conditions of adolescents through modifying the exercise environment and participating in after-school exercise interventions. Although further prospective studies are needed, these factors should be taken into consideration when discussing and planning exercise intervention programs for adolescents. In Taiwan, most adolescents prioritize academic work over exercise participation. Therefore, further studies should focus on how after-school exercise can be made to appear more attractive and worthwhile to encourage school-age youth to participate in exercise.

Acknowledgments: This study is based in part on data from the National Physical Fitness Examination Survey Database provided by the Sports and Health Information Application Research Center, Sport Administration, Ministry of Education. The interpretation and conclusions contained herein do not represent those of Sport Administration, Ministry of Education.

Author Contributions: Chien-Chang Ho participated in the design, conducted the statistical analyses, interpreted the data, and drafted the manuscript; Kai-Yang Lo supervised the study, assisted in data interpretation, and critically reviewed the manuscript; Min-Chen Wu and Shu-Chin Tung helped conduct the study and revise the manuscript; City C. Hsieh and Hsueh-Hua Yao helped to manage and analyze the data. All authors read and approved the final manuscript.

Conflicts of Interest: The authors declare no conflict of interest.

\section{Abbreviations}

The following abbreviations are used in this manuscript:

ANOVA Analysis of variance

BMI Body mass index

NCPFS National Council on Physical Fitness and Sports

NPFS National Physical Fitness Survey

SD Standard deviation 


\section{References}

1. Andersen, L.B.; Harro, M.; Sardinha, L.B.; Froberg, K.; Ekelund, U.; Brage, S.; Anderssen, S.A. Physical activity and clustered cardiovascular risk in children: A cross-sectional study (The European Youth Heart Study). Lancet 2006, 368, 299-304. [CrossRef]

2. Biddle, S.J.; Gorely, T.; Stensel, D.J. Health-enhancing physical activity and sedentary behaviour in children and adolescents. J. Sports Sci. 2004, 22, 679-701. [CrossRef] [PubMed]

3. Steinberger, J.; Daniels, S.R. Obesity, insulin resistance, diabetes, and cardiovascular risk in children: An American Heart Association scientific statement from the Atherosclerosis, Hypertension, and Obesity in the Young Committee (Council on Cardiovascular Disease in the Young) and the Diabetes Committee (Council on Nutrition, Physical Activity, and Metabolism). Circulation 2003, 107, 1448-1453. [PubMed]

4. Tsai, C.C.; Chen, L.H.; Wong, J.M. Physical activity and health related physical fitness in Taiwanese. Bull. Phys. Educ. 1998, 26, 153-160.

5. Department of Health. Taiwan Youth Health Survey Report; Executive Yuen, Department of Health: Taipei, Taiwan, 2006.

6. Bauman, A.E.; Reis, R.S.; Sallis, J.F.; Wells, J.C.; Loos, R.J.; Martin, B.W. Correlates of physical activity: Why are some people physically active and others not? Lancet 2012, 380, 258-271. [CrossRef]

7. Caspersen, C.J.; Powell, K.E.; Christenson, G.M. Physical activity, exercise, and physical fitness: Definitions and distinctions for health-related research. Public Health Rep. 1985, 100, 126-131. [PubMed]

8. Hsieh, P.L.; Chen, M.L.; Huang, C.M.; Chen, W.C.; Li, C.H.; Chang, L.C. Physical activity, body mass index, and cardiorespiratory fitness among school children in Taiwan: A cross-sectional study. Int. J. Environ. Res. Public Health 2014, 11, 7275-7285. [CrossRef] [PubMed]

9. Grund, A.; Dilba, B.; Forberger, K.; Krause, H.; Siewers, M.; Rieckert, H.; Müller, M.J. Relationships between physical activity, physical fitness, muscle strength and nutritional state in 5-to 11-year-old children. Eur. J. Appl. Physiol. 2000, 82, 425-438. [CrossRef] [PubMed]

10. Chen, L.J.; Fox, K.R.; Haase, A.M. Body shape dissatisfaction and obesity among Taiwanese adolescents. Asia Pac. J. Clin. Nutr. 2008, 17, 457-460. [PubMed]

11. Chen, W.; Lin, C.C.; Peng, C.T.; Li, C.I.; Wu, H.C.; Chiang, J.; Wu, J.Y.; Huang, P.C. Approaching healthy body mass index norms for children and adolescents from health-related hysical fitness. Obes. Rev. 2003, 3, 225-232. [CrossRef]

12. Huang, Y.C.; Malina, R.M. BMI and health-related physical fitness in Taiwanese youth 9-18 years. Med. Sci. Sports Exerc. 2007, 39, 701-708. [CrossRef] [PubMed]

13. Liu, Y.S.; Hsieh, S.S. Effects of single bout long duration and multiple bouts short duration exercise on energy expenditure. Med. Sci. Sports Exerc. 2004, 36, 1897.

14. Chen, L.J.; Fox, K.R.; Haase, A.; Wang, J.M. Obesity, fitness and health in Taiwanese children and adolescents. Eur. J. Clin. Nutr. 2006, 60, 1367-1375. [CrossRef] [PubMed]

15. Fernandes, M.; Sturm, R. Facility provision in elementary schools: Correlates with physical education, recess, and obesity. Prev. Med. 2010, 50, 30-35. [CrossRef] [PubMed]

16. Mota, J.; Santos, M.P.; Ribeiro, J.C. Differences in leisure-time activities according to level of physical activity in adolescents. J. Phys. Act. Health 2008, 5, 286-293. [CrossRef] [PubMed]

17. Pelclová, J.; El Ansari, W.; Vasícková, J. Is participation in after-school physical activity associated with increased total physical activity? A study of high school pupils in the Czech Republic. Int. J. Environ. Res. Public Health. 2010, 7, 2853-2865. [CrossRef] [PubMed]

18. Ministry of Education. Physical Fitness Measurement; Sports Administration, Ministry of Education: Taipei, Taiwan, 2006.

19. Lu, C.M.; Lee, M.S.; Chen, S.C. Determinants of physical activity in urban primary school children. J. Health Educ. 2000, 14, 95-111.

20. Wu, S.Y.; Pender, N.; Noureddine, S. Gender differences in the psychosocial and cognitive correlates of physical activity among Taiwanese adolescents: A structural equation modeling approach. Int. J. Behav. Med. 2003, 10, 93-105. [CrossRef] [PubMed]

21. Lu, L. The effects of physical activity in health-related physical fitness in elementary school-age children. Bull. Phys. Educ. 1998, 20, 433-442.

22. Wu, Y.J.; Smith, D.E. Self-esteem of Taiwanese children. Child Study J. 1997, 27, 1-19. 
23. Li, C.W.; Fang, C.L. Factors related to middle school children's physical activity and fitness. Bull. Phys. Educ. 1998, 26, 139-148.

24. Huang, Y.C.; Lin, T.C.; Kao, Y.K. Relationship between goal orientation and moderate-to-vigorous physical activity in school children: Sex, grade and racial variation. Bull. Phys. Educ. 2000, 29, 71-80.

25. Chen, L.J.; Haase, A.M.; Fox, K.R. Physical activity among adolescents in Taiwan. Asia Pac. J. Clin. Nutr. 2007, 16, 354-361. [PubMed]

26. Huang, S.J.; Hung, W.C.; Sharpe, P.A.; Wai, J.P. Neighborhood environment and physical activity among urban and rural schoolchildren in Taiwan. Health Place 2010, 16, 470-476. [PubMed]

27. Chen, H. Comparison of physical fitness and fundamental ability of exercise between Han children and native school children in Taiwan. Bull. Taichung Teach. Coll. 1995, 9, 577-609.

28. Shen, C.K.; Huang, J. The relationship between activity space and health-related fitness in primary school students. Bull. Phys. Educ. 2001, 31, 81-90.

29. Chen, J.L.; Unnithan, V.; Kennedy, C.; Yeh, C.H. Correlates of physical fitness and activity in Taiwanese children. Int. Nurs. Rev. 2008, 55, 81-88. [CrossRef] [PubMed]

30. Chen, W.S.; Leong, T.C. Differences in health-related physical fitness in elementary school-age students between rural and urban schools. Bull. Phys. Educ. Coll. 1995, 3, 129-145.

31. Lee, S.S. Case study of health-related physical fitness on primary school children aged 9-12. Peng Tung Bull. Educ. 1996, 9, 551-584.

32. Felton, G.M.; Dowda, M.; Ward, D.S.; Dishman, R.K.; Trost, S.G.; Saunders, R.; Pate, R.R. Differences in physical activity between black and white girls living in rural and urban areas. J. Sch. Health 2002, 72, 250-255. [CrossRef] [PubMed]

33. Loucaides, C.A.; Plotnikoff, R.C.; Bercovitz, K. Differences in the correlates of physical activity between urban and rural Canadian youth. J. Sch. Health 2007, 77, 164-170. [CrossRef] [PubMed]

34. Tudor-Locke, C.; Ainsworth, B.E.; Popkin, B.M. Active commuting to school: An overlooked source of childrens' physical activity? Sports Med. 2001, 31, 309-313. [CrossRef] [PubMed]

(C) 2017 by the authors; licensee MDPI, Basel, Switzerland. This article is an open access article distributed under the terms and conditions of the Creative Commons Attribution (CC-BY) license (http:/ / creativecommons.org/licenses/by/4.0/). 